\title{
Site-dependent differential KIT and PDGFRA expression in gastric and intestinal gastrointestinal stromal tumors
}

\author{
Florian Haller ${ }^{1}$, Nicole Happel ${ }^{2}$, Hans-Jürgen Schulten ${ }^{1}$, Anja von Heydebreck ${ }^{3}$, \\ Stefanie Schwager ${ }^{1}$, Thomas Armbrust ${ }^{4}$, Claus Langer ${ }^{5}$, Bastian Gunawan ${ }^{1}$, \\ Detlef Doenecke ${ }^{2}$ and László Füzesi ${ }^{1}$
}

${ }^{1}$ Department of Pathology, Georg-August University, Göttingen, Germany; ${ }^{2}$ Institute for Biochemistry and Molecular Cell Biology, Georg-August University, Göttingen, Germany; ${ }^{3}$ Department of Bio- and Chemoinformatics, Merck KGaA, Darmstadt, Germany; ${ }^{4}$ Department of Gastroenterology and Endocrinology, Georg-August University, Göttingen, Germany and ${ }^{5}$ Department of General Surgery, Georg-August University, Göttingen, Germany

\begin{abstract}
In gastrointestinal stromal tumors (GISTs), mutually exclusive gain-of-function mutations of KIT and PDGFRA are associated with different mutation-dependent clinical behavior. Taking into account the well-known different clinical behavior of GISTs from the stomach or the intestine, the aim of the current study is to evaluate the mutation- and site-dependent effects on mRNA and protein expression of $K I T$ and PDGFRA in a large series of primary GISTs. Fresh-frozen tissue of 53 primary GISTs from gastric $(75 \%)$ or intestinal $(25 \%)$ sites were analyzed for mutation of KIT or PDGFRA using direct sequencing. Furthermore, KIT and PDGFRA mRNA and protein expression were determined using quantitative RT-PCR and quantitative densitometric evaluation of Western blot data. Each tumor either had a mutation of KIT (79\%) or PDGFRA (21\%). All GISTs with PDGFRA mutation were from gastric sites. Mutation-dependently, GISTs with KIT mutation had a significantly higher expression of $K I T$ and at the same time a significantly lower expression of PDGFRA compared to GISTs with PDGFRA mutation. Site-dependently, gastric GISTs had a significantly higher expression of PDGFRA and a significantly lower expression of KIT compared to intestinal GISTs. Additionally, even if the KIT-mutated GISTs alone were considered, a significantly higher expression of PDGFRA could be observed in gastric than in intestinal tumors. We also found a significant correlation between a higher protein expression of PDGFRA and longer disease-free survival. The correlation of gastric site and PDGFRA mutation with higher PDGFRA expression and longer disease-free survival suggests different regulatory roles of KIT and PDGFRA gene expression on the control of cell proliferation, and, thereby on clinical behavior. The higher PDGFRA expression in gastric GISTs possibly contributes to the well-known site-dependent clinical behavior. Modern Pathology (2007) 20, 1103-1111; doi:10.1038/modpathol.3800947; published online 3 August 2007
\end{abstract}

Keywords: KIT; PDGFRA; GIST; mRNA expression; protein expression; qRT-PCR

Gain-of-function mutations of the type III receptor tyrosine kinases KIT (v-kit Hardy-Zuckerman 4 feline sarcoma viral oncogene homolog) and PDGFRA (platelet-derived growth factor receptor, $\alpha$ polypeptide) have been identified as the central event in the tumorigenesis of gastrointestinal stromal tumors (GISTs). ${ }^{1,2}$ The interstitial cells of Cajal located in the muscular layer of the gastrointestinal

Correspondence: Dr F Haller, MD, Department of Pathology, University of Göttingen, Robert-Koch-Str. 40, Göttingen D-37099, Germany.

E-mail: florian.haller@med.uni-goettingen.de

Received 23 May 2007; revised 6 July 2007; accepted 9 July 2007; published online 3 August 2007 tract are regarded as the precursor cells for GISTs, ${ }^{3}$ and ligand-independent constitutive receptor tyrosine kinase signaling presumably initiates neoplastic formation in interstitial cells of Cajal through elevated cell proliferation. ${ }^{4}$ Mutations of KIT and PDGFRA have been demonstrated to occur mutually exclusively. About $60-70 \%$ of GISTs have activating mutations of KIT, whereas mutations of PDGFRA are less frequent and can be found in only $10-20 \%$ of GISTs. $^{5-9}$ GISTs with KIT mutation and PDGFRA mutation are characterized by distinct histomorphologic phenotypes, and GISTs with PDGFRA mutation appear clinically less aggressive. ${ }^{9-15}$ Two similar studies analyzing gene expression in GISTs with KIT or PDGFRA mutation reported that the 
mRNA and protein expression of KIT and PDGFRA are highly different between these two types of tumors. ${ }^{16,17}$ Although both genes were basically expressed in all tumors, the quantitative regulation was different, in that tumors with KIT mutation had a higher expression of KIT, whereas the tumors with PDGFRA mutation had a higher expression of PDGFRA. These findings suggest an important impact of the activating mutation on the expression of the mutated gene itself, providing a possible explanation for mutation-dependent differences in clinical behavior.

It has also been observed that GISTs from the intestine generally exhibit a more aggressive clinical course compared to GISTs from the stomach. ${ }^{18,19}$ Correspondingly, Antonescu et $a l^{20}$ found an anatomical site-dependent gene expression in GISTs, and PDGFRA was among the genes that were expressed higher in the gastric tumors.

The aim of the current study was to determine the mRNA and protein expression of KIT and PDGFRA in a large series of 53 primary GISTs with mutation of KIT or PDGFRA from different anatomical sites, to evaluate quantitatively site-dependent differences in the expression levels of KIT and PDGFRA. Furthermore, we compared the impact of mutated gene, anatomical site and gene expression on the clinical behavior, to determine whether the expression of KIT and PDGFRA would be a valuable independent factor for the prognosis of GISTs.

\section{Materials and methods}

\section{Tumor Samples}

This study comprises both snap-frozen as well as formalin-fixed and paraffin-embedded tissue samples from 53 primary GISTs. All tumors were removed surgically without other prior treatment. None of the patients (Table 1) had received imatinib mesylate (Gleevec, Novartis, Switzerland) before operation or disease progression. No adjuvant treatment has been carried out. Mitoses were counted in 50 high-power fields (HPFs), while proliferation rate was estimated as the percentage of Mib1-positive nuclei from tumor areas with the highest mitotic activity. Risk of clinically aggressive behavior was evaluated according to the consensus approach published by Fletcher et $a{ }^{21}{ }^{21}$ and the malignant potential was evaluated according to the proposal of Miettinen et al. ${ }^{22}$

\section{Mutation Analysis of KIT and PDGFRA Genes}

Mutation analysis of KIT exons 9, 11, 13 and 17, as well as PDGFRA exons 12,14 and 18 was performed on snap-frozen tumor tissue from all 53 primary GISTs using direct sequencing of PCR products as described previously. ${ }^{23}$

\section{RNA Isolation and Reverse Transcription}

Total RNA was isolated from snap-frozen tumor tissue from all 53 GISTs using $\sim 50 \mathrm{mg}$ frozen tissue per ml TRIzol (Invitrogen Life Technologies, Karlsruhe, Germany) as described previously. ${ }^{23}$ Total RNA concentration was quantified with the RNA 6000 nano LabChip using the Agilent 2100 Bioanalyzer (Agilent Technologies, Palo Alto, CA, USA).

\section{Quantitative PCR}

Gene specific primers for KIT (5'-ATGTTGTCCCAA CCAAGG-3' and $5^{\prime}$-CTTCTAAGTCTAGGGCCAAC TC-3') and PDGFRA (5'-TGTCCTGGTTGTCATTTG GA-3' and 5'-CTTCAACCACCTTCCCAAAC- ${ }^{\prime}$ ) were designed on different exons with a melting temperature of $60^{\circ} \mathrm{C}$ for PCR products with a length of 114 and 200 nucleotide pairs, respectively. PCR was run in $20 \mu \mathrm{l}$ reactions in triplicates on an iCycler (Bio-Rad Laboratories GmbH, München, Germany) using the QuantiTect SYBR Green PCR Kit (Qiagen, Hilden, Germany) and gene-specific primers in a final concentration of $300 \mathrm{nM}$. The temperature profile consisted of (i) an initial step at $95^{\circ} \mathrm{C}$ for $10 \mathrm{~s}$ for Taq activation; (ii) 40 cycles at $95^{\circ} \mathrm{C}$ for $15 \mathrm{~s}$ and $60^{\circ} \mathrm{C}$ for $60 \mathrm{~s}$; and (iii) a final melting curve analysis with a temperature ramp from 60 to $95^{\circ} \mathrm{C}$ and a heating rate of $3^{\circ} \mathrm{C} / \mathrm{min}$. PCR efficiencies were calculated with a relative standard curve derived from a cDNA mixture (a twofold dilution series with seven measuring points in triplicates) and gave regression coefficients $>0.95$ and reproducible primer-specific efficiencies of 85-99\%. Gene-specific amplification was confirmed by a single peak in melting curve analysis and a single band in highresolution agarose gel electrophoresis (SeaKem LE agarose, BMA Rockland, ME, USA). No template controls (no cDNA in PCR) and genomic controls (no enzyme in reverse transcription reaction) were run for each gene to detect unspecific and genomic amplification or primer dimerization. Relative expression levels were calculated from the relative standard curve. ${ }^{24}$ The reference genes $18 \mathrm{~S}$ rRNA and actin beta (ACTB) could be confirmed to be equivalently expressed within most of the examined groups as described previously. ${ }^{25}$ Expression of KIT and PDGFRA mRNA was calculated in relation to the mean expression of $18 \mathrm{~S}$ and $A C T B$, and logarithmized to obtain approximately normally distributed data. The fold-change comparisons for the expression of KIT and PDGFRA between different groups were calculated as the ratio between the means.

\section{SDS-PAGE and Western Blot Analysis}

Total lysates of 49 snap-frozen tumor tissues were separated on 10 or $12 \%$ SDS-polyacrylamide gels (SDS-PAGE), and the proteins were electrophoretically 
Table 1 Clinicopathologic data in 53 primary GISTs

\begin{tabular}{|c|c|c|c|c|c|c|c|}
\hline Patient no. & Age/gender & Site & Mutation & $\begin{array}{l}\text { Tumor diameter } \\
(\mathrm{cm})\end{array}$ & $\begin{array}{l}\text { Mitotic count/ } \\
\text { 50HPFs }\end{array}$ & $\begin{array}{c}\text { DFS } \\
\text { (months) }\end{array}$ & $\begin{array}{l}\text { OS } \\
\text { (months) }\end{array}$ \\
\hline 1 & $45 / F$ & Gastric & PDGFRA D842V & $<5$ & $<5$ & 104 & NED 104 \\
\hline 2 & $84 / \mathrm{M}$ & Gastric & PDGFRA D $842 \mathrm{~V}$ & $<5$ & $<5$ & 11 & NED 11 \\
\hline 3 & $53 / \mathrm{F}$ & Gastric & PDGFRA D842V & $<5$ & $<5$ & 9 & NED 9 \\
\hline 4 & $61 / \mathrm{M}$ & Gastric & PDGFRA Dup 556-565 & $<5$ & $<5$ & 40 & NED 40 \\
\hline 5 & $69 / \mathrm{F}$ & Gastric & PDGFRA D842V & $<5$ & $<10$ & 19 & NED 19 \\
\hline 6 & $66 / \mathrm{M}$ & Gastric & PDGFRA D842H, Del 843-846 & $<5$ & $>10$ & 69 & NED 69 \\
\hline 7 & $53 / \mathrm{M}$ & Gastric & PDGFRA D842A, Del 843-846 & $<10$ & $<5$ & 81 & NED 81 \\
\hline 8 & $58 / \mathrm{M}$ & Gastric & PDGFRA D842V & $<10$ & $<5$ & 19 & NED 19 \\
\hline 9 & $65 / \mathrm{M}$ & Gastric & PDGFRA D $842 \mathrm{~V}$ & $<10$ & $<5$ & 64 & NED 64 \\
\hline 10 & $66 / \mathrm{M}$ & Gastric & PDGFRA D842V & $<10$ & $<5$ & 29 & NED 29 \\
\hline 11 & $66 / \mathrm{M}$ & Gastric & PDGFRA D842V & $<10$ & $<5$ & 50 & NED 50 \\
\hline 12 & $66 / \mathrm{M}$ & Gastric & KIT Del 553-558 & $<5$ & $<5$ & 12 & NED 12 \\
\hline 13 & $77 / \mathrm{F}$ & Gastric & KIT Del 557 & $<5$ & $<5$ & 9 & DOO 9 \\
\hline 14 & $78 / \mathrm{F}$ & Gastric & KIT Del 560 & $<5$ & $<5$ & 14 & NED 14 \\
\hline 15 & $52 / \mathrm{M}$ & Gastric & KIT K550L, Del551-556 & $<5$ & $<5$ & 28 & NED 28 \\
\hline 16 & $80 / \mathrm{F}$ & Gastric & KIT V559D & $<5$ & $<5$ & 62 & NED 62 \\
\hline 17 & $69 / \mathrm{M}$ & Gastric & KIT V559D & $<5$ & $<5$ & 8 & NED 8 \\
\hline 18 & $73 / \mathrm{M}$ & Gastric & KIT V560D & $<5$ & $<5$ & 90 & NED 90 \\
\hline 19 & $79 / F$ & Gastric & KIT V560D & $<5$ & $<5$ & 58 & DOO 58 \\
\hline 20 & $52 / \mathrm{M}$ & Gastric & KIT W557E, Del 558 & $<5$ & $<5$ & 26 & NED 26 \\
\hline 21 & $86 / F$ & Gastric & KIT W557R & $<5$ & $<5$ & 28 & NED 28 \\
\hline 22 & $82 / \mathrm{F}$ & Gastric & KIT Del 557-558 & $<5$ & $>10$ & 57 & NED 57 \\
\hline 23 & $72 / \mathrm{F}$ & Gastric & KIT Del 558-562 & $<5$ & $>10$ & 72 & NED 72 \\
\hline 24 & $53 / \mathrm{M}$ & Gastric & KIT Del 557-558 & $<10$ & $<5$ & 18 & NED 18 \\
\hline 25 & $69 / \mathrm{M}$ & Gastric & KIT Del 557-558 & $<10$ & $<5$ & 11 & NED 11 \\
\hline 26 & $67 / \mathrm{M}$ & Gastric & KIT Dup 577-580 & $<10$ & $<5$ & 51 & NED 51 \\
\hline 27 & $70 / \mathrm{F}$ & Gastric & KIT V559D & $<10$ & $<5$ & 112 & NED 112 \\
\hline 28 & $47 / \mathrm{M}$ & Gastric & KIT V559G & $<10$ & $<5$ & 78 & NED 78 \\
\hline 29 & $85 / \mathrm{F}$ & Gastric & KIT V560D & $<10$ & $<5$ & 35 & NED 35 \\
\hline 30 & $54 / \mathrm{M}$ & Gastric & KIT V560D & $<10$ & $<5$ & 0 & AWD 14 \\
\hline 31 & $64 / \mathrm{F}$ & Gastric & KIT Del 552-555 & $<10$ & $<10$ & 92 & NED 92 \\
\hline 32 & $64 / \mathrm{M}$ & Gastric & KIT Del 555-559 & $<10$ & $>10$ & 39 & NED 39 \\
\hline 33 & $58 / \mathrm{M}$ & Gastric & KIT Del 557-558 & $<10$ & $>10$ & 34 & AWD 72 \\
\hline 34 & $72 / \mathrm{M}$ & Gastric & KIT Dup 502-503 & $<10$ & $>10$ & 25 & NED 25 \\
\hline 35 & $49 / \mathrm{F}$ & Gastric & KIT Dup 571-579 & $>10$ & $<5$ & 1 & AWD 1 \\
\hline 36 & $78 / \mathrm{F}$ & Gastric & KIT Dup 502-503 & $>10$ & $<10$ & 65 & NED 65 \\
\hline 37 & $68 / \mathrm{M}$ & Gastric & KIT Del $550-558^{\mathrm{a}}$ & $>10$ & $>10$ & 38 & NED 38 \\
\hline 38 & $63 / \mathrm{M}$ & Gastric & KIT K558R, Del 559-565 & $>10$ & $>10$ & 17 & AWD 54 \\
\hline 39 & $64 / \mathrm{M}$ & Gastric & KIT V559D & $>10$ & $>10$ & 84 & AWD 111 \\
\hline 40 & $61 / \mathrm{F}$ & Gastric & KIT W557C, Del 558-559 & $>10$ & $>10$ & 23 & AWD 78 \\
\hline 41 & $68 / \mathrm{F}$ & Intestinal & KIT Del 555-556 & $<5$ & $<5$ & 21 & NED 21 \\
\hline 42 & $54 / \mathrm{F}$ & Intestinal & KIT Del 557-558 & $<10$ & $<5$ & 19 & NED 19 \\
\hline 43 & $82 / \mathrm{M}$ & Intestinal & KIT V559D & $<10$ & $<5$ & 42 & NED 42 \\
\hline 44 & $54 / \mathrm{M}$ & Intestinal & KIT V559G & $<10$ & $<5$ & 62 & NED 62 \\
\hline 45 & $74 / \mathrm{F}$ & Intestinal & KIT Dup 502-503 & $<10$ & $<10$ & 13 & NED 13 \\
\hline 46 & $67 / \mathrm{M}$ & Intestinal & $\begin{array}{l}\text { KIT K558N, Del } 559 \\
\text { (hemizygot) }\end{array}$ & $<10$ & $<10$ & 41 & AWD 46 \\
\hline 47 & $56 / \mathrm{M}$ & Intestinal & KIT Del 556-558 & $<10$ & $>10$ & 0 & DOTD 19 \\
\hline 48 & $62 / \mathrm{F}$ & Intestinal & KIT Del 557-558 & $<10$ & $>10$ & 0 & AWD 47 \\
\hline 49 & $54 / \mathrm{F}$ & Intestinal & KIT Del 559-561 & $<10$ & $>10$ & 0 & AWD 36 \\
\hline 50 & $43 / \mathrm{M}$ & Intestinal & KIT K558N, Del 559 & $<10$ & $>10$ & 13 & AWD 76 \\
\hline 51 & $61 / \mathrm{M}$ & Intestinal & KIT V559D & $<10$ & $>10$ & 19 & AWD 95 \\
\hline 52 & $73 / \mathrm{M}$ & Intestinal & KIT W557G & $<10$ & $>10$ & 0 & AWD 26 \\
\hline 53 & $57 / \mathrm{M}$ & Intestinal & KIT V560E & $>10$ & $<5$ & 86 & NED 86 \\
\hline
\end{tabular}

AWD, alive with disease; DFS, disease-free survival; DOO, died of other causes; DOTD, died of tumor disease; F, female; GIST, gastrointestinal stromal tumor; M, male; NED, no evidence of disease; OS, overall survival.

a additional KIT exon 9 del C1461 in codon 480.

transferred to nitrocellulose filters. Uniform blotting was checked by staining the nitrocellulose filter with Ponceau S (Sigma, Taufkirchen, Germany). Blots were cut horizontally at the $72-\mathrm{kDa}$ marker band. The upper share of the blots were probed with anti-KIT antibody (no. 3392; Cell Signalling
Technology, New England Biolabs, Frankfurt, Germany) or anti-PDGFRA antibody (no. 3164; Cell Signalling Technology), and the lower share with an anti- $\beta$-actin antibody (no. 4967; Cell Signalling Technology) in a 1:1000 dilution. The immunoreactive proteins were visualized using the 
chemiluminescence ECL plus detection system (Amersham Bioscience, Freiburg, Germany) after incubation with a horseradish peroxidase-conjugated goat anti-rabbit antibody (no. A0545; Sigma). Stripping of blotting membranes for reprobing was carried out by incubation of the membranes in stripping buffer $(100 \mathrm{mM}$ 2-mercaptoethanol, $2 \%$ SDS, $62.5 \mathrm{mM}$ Tris-HCl, $\mathrm{pH}$ 6.7) for $20 \mathrm{~min}$ at $60^{\circ} \mathrm{C}$ with occasional agitation. Bands on the ECL film were quantified using the 1Dscan Ex gel analysis software (Scanalytics, Rockville, MD, USA). The different Western blots were adjusted by the presence of one calibrator sample on every single gel. The raw densitometric intensities for KIT and PDGFRA varied between 2 and 15000 for the different tumor samples. In those cases where the protein amount was under the detection limit, a value of 1 was assigned. Relative protein amounts of KIT and PDGFRA were calculated in relation to $\beta$ actin, and logarithmized to obtain approximately normally distributed data. The fold-change comparisons for the expression of KIT and PDGFRA between different groups were calculated as the ratio between the means.

\section{Statistics}

Descriptive statistics, tests and graphs were performed with Statistica 6.0 (StatSoft, Hamburg, Germany), and the statistical software system R. ${ }^{26}$ Associations between the clinicopathological and the molecular genetic parameters were evaluated using the Wilcoxon test or the Fisher test in the case of categorical variables. Disease-free survival rates were plotted by the Kaplan-Meier method. Associations of patient and tumor parameters with diseasefree survival times were assessed using the log-rank test.

\section{Results}

\section{Clinicopathologic Analysis and Disease-Free Survival}

The current study comprised 53 primary GISTs, including 40 (75\%) from gastric and 13 (25\%) from intestinal sites (Table 1). Mutation analysis revealed KIT mutation in $42(79 \%)$ cases and PDGFRA mutation in $11(21 \%)$ cases. No cases were observed with both KIT and PDGFRA mutation. All 11 cases with PDGFRA mutation were from gastric sites, while $29(69 \%)$ and $13(31 \%)$ of the cases with KIT mutation were from gastric and intestinal sites, respectively $(P=0.03)$. Six $(11 \%)$ patients had synchronous metastatic disease at the time of operation. Seven (13\%) patients developed metachronous progressive disease after a mean of 33 months (range: $13-84$ months). Forty (75\%) patients had no disease progression after a mean follow-up of 44 months (range: 8-112 months). Patients with gastric tumors had significantly longer disease-free

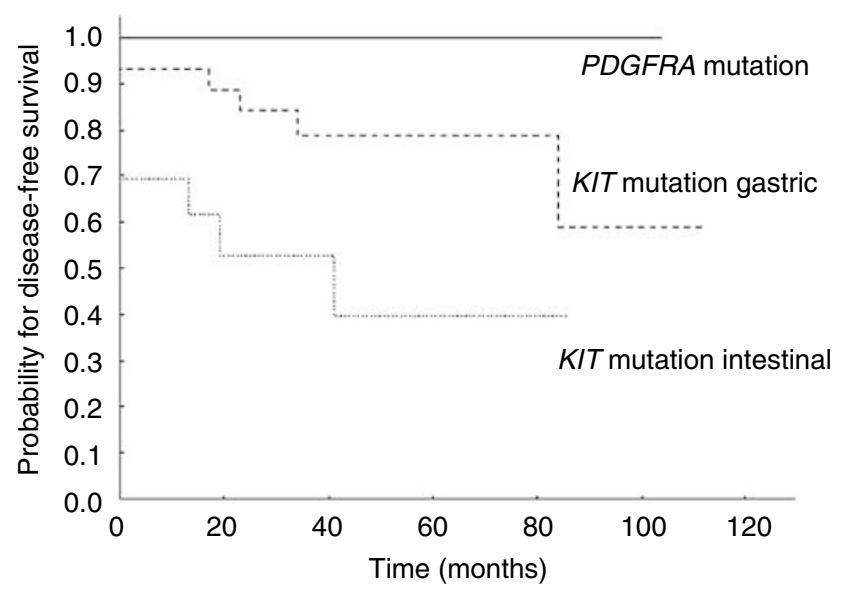

Figure 1 Mutation- and site-dependent differences in disease-free survival. PDGFRA-mutated GISTs were exclusively from gastric sites, and none of these patients had tumor progress. Patients with gastric KIT-mutated tumors had significantly longer disease-free survival compared to patients with intestinal KIT-mutated tumors $(P=0.03)$. There was no statistically significant difference in disease-free survival for patients with PDGFRA-mutated tumors and gastric KIT-mutated tumors.

survival than patients with intestinal tumors $(P=0.004)$, and patients with PDGFRA-mutated tumors had a significantly longer disease-free survival than patients with KIT-mutated tumors $(P=0.04)$. Additionally, KIT-mutated tumors from gastric sites were associated with significantly longer disease-free survival compared to KIT-mutated tumors from intestinal sites $(P=0.03$, Figure 1). On the other hand, there was no significant difference in disease-free survival when comparing gastric tumors with PDGFRA and KIT mutation.

\section{Mutation-Dependent Expression of KIT and PDGFRA}

The mean KIT expression was 10.3-fold higher on the mRNA level $\left(P=6 \times 10^{-7}\right)$ and 7.5 -fold higher on the protein level $(P=0.03)$ in both gastric and intestinal GISTs with KIT mutation compared to GISTs with PDGFRA mutation (Figure 2a and b). On the other hand, the mean PDGFRA expression was 7.3-fold lower on the mRNA level $\left(P=5 \times 10^{-4}\right)$ and 25.7 -fold lower on the protein level $(P=0.001)$ in GISTs with KIT mutation (Figure 2c and d).

\section{Site-Dependent Expression of KIT and PDGFRA}

In addition to the mutation-dependent expression of KIT and PDGFRA, the expression of these two genes was also site-dependent. The mean PDGFRA expression was significantly higher in gastric GISTs compared to their intestinal counterparts (8.2-fold higher on the mRNA level, $P=8 \times 10^{-4}$; and 86.2fold higher on the protein level, $P=3 \times 10^{-5}$, Figure 3a and b). In contrast, the mean KIT expression was significantly lower in gastric GISTs (2.6-fold lower 
a

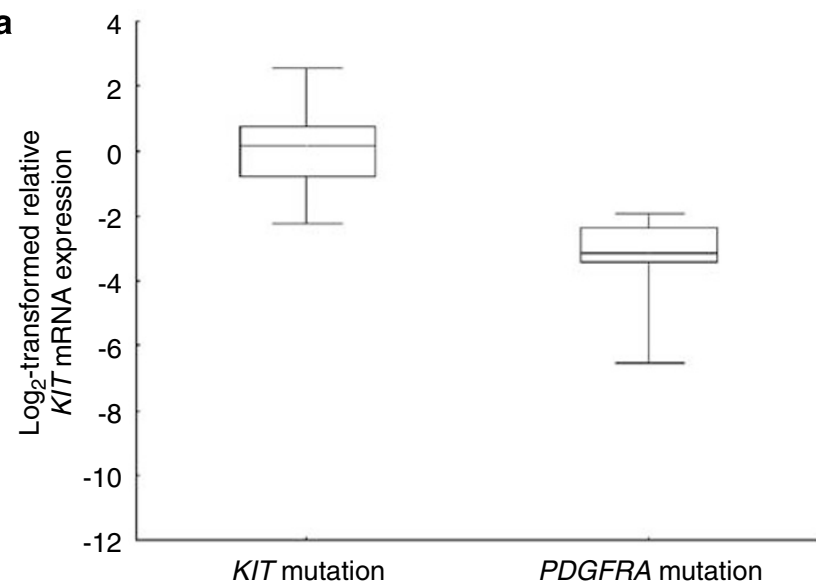

c
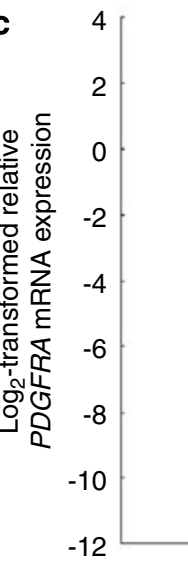

KIT mutation

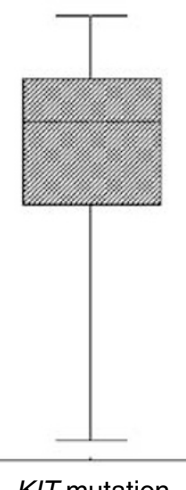

KIT mutation b

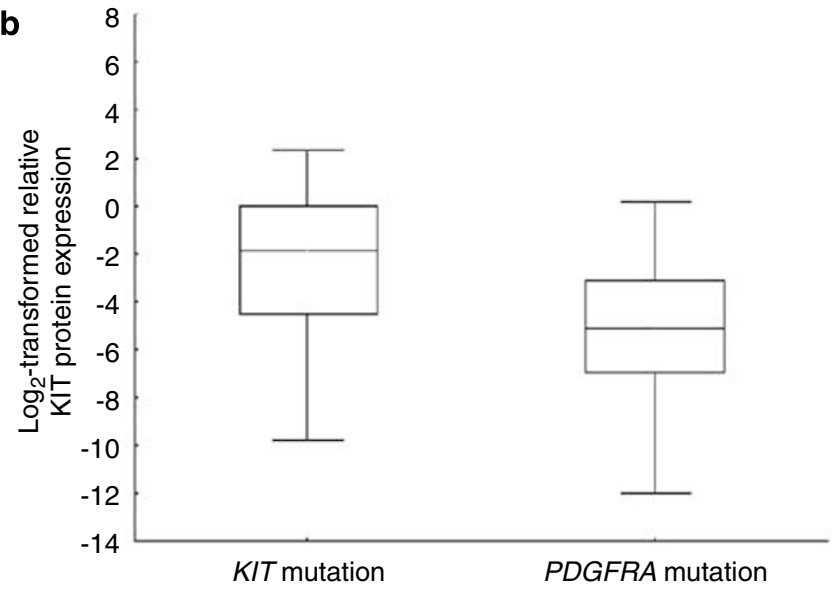

d

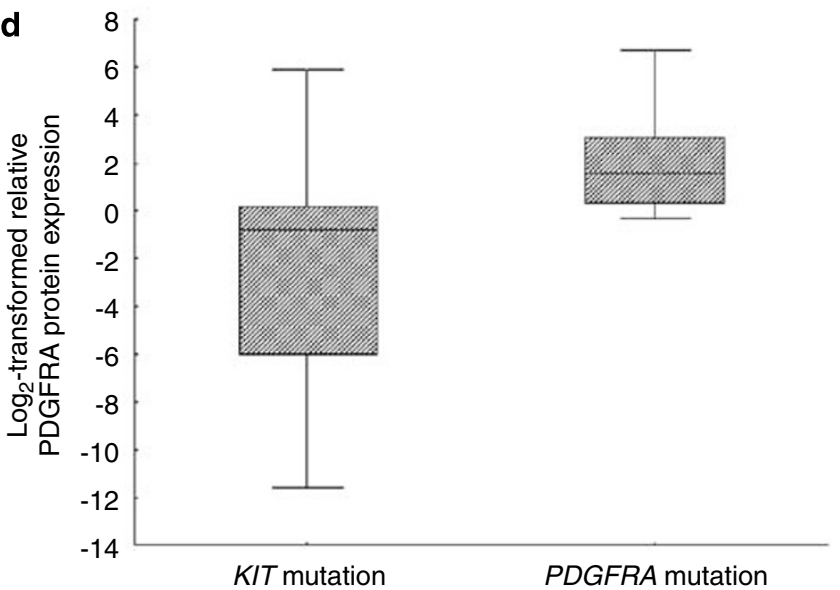

Figure 2 Mutation-dependent mRNA and protein expression of KIT and PDGFRA. (a) KIT mRNA expression; (b) KIT protein expression; (c) PDGFRA mRNA expression; and (d) PDGFRA protein expression. KIT mRNA and protein expression (open box) were significantly higher in GISTs with KIT mutation. PDGFRA mRNA and protein expression (hatched box) were significantly higher in GISTs with PDGFRA mutation. Horizontal line, median; inner box, 25 and 75\% interval; outer spread, minimum and maximum.

on the mRNA level, $P=0.02$; and 5.3-fold lower on the protein level, $P=0.006$, Figure $3 \mathrm{c}$ and d).

This mutation-independent but site-dependent difference was even more obvious when only KITmutated GISTs from gastric and intestinal sites were compared. Gastric GISTs with KIT mutation expressed PDGFRA 5.5-fold higher on the mRNA level $(P=0.008)$ and 52.2 -fold higher on the protein level $\left(P=2 \times 10^{-4}\right)$ compared to their intestinal counterparts with KIT mutation (Figure 4a and b). On the other hand, although the mean mRNA expression of KIT was not significantly different between gastric and intestinal GISTs with KIT mutation (Figure 4c), the mean protein expression of KIT was 3.7-fold lower in gastric GISTs with KIT mutation compared to intestinal GISTs with KIT mutation $(P=0.02$, Figure 4d). The comparison of mutation-dependent and site-dependent regulation of KIT and PDGFRA expression revealed that the regulation of PDGFRA expression was equally mutation- and site-dependent, whereas the regulation of KIT expression was rather mutation-dependent (Figure 4).

\section{Expression of PDGFRA and Disease-Free Survival}

A significantly higher mean expression of PDGFRA was detected in the 40 GISTs without tumor progress than in the 13 GISTs with tumor progress (2.8-fold higher on the mRNA level, $P=0.06$ and 27.3-fold higher on the protein level, $P=0.002$ ). Correspondingly, a two-sided group-wise comparison of tumors with high vs low PDGFRA protein expression separated by the mean revealed that higher PDGFRA protein expression significantly correlated with longer disease-free survival $(P=0.01$, Figure 5).

\section{Discussion}

Apart from the previously described mutationdependent expression of KIT and PDGFRA, ${ }^{16,17}$ we report here on a site-dependent expression of KIT and PDGFRA. Gastric GISTs had a 8.2-fold higher mRNA expression and a 86.2-fold higher protein expression of PDGFRA compared to GISTs from the 

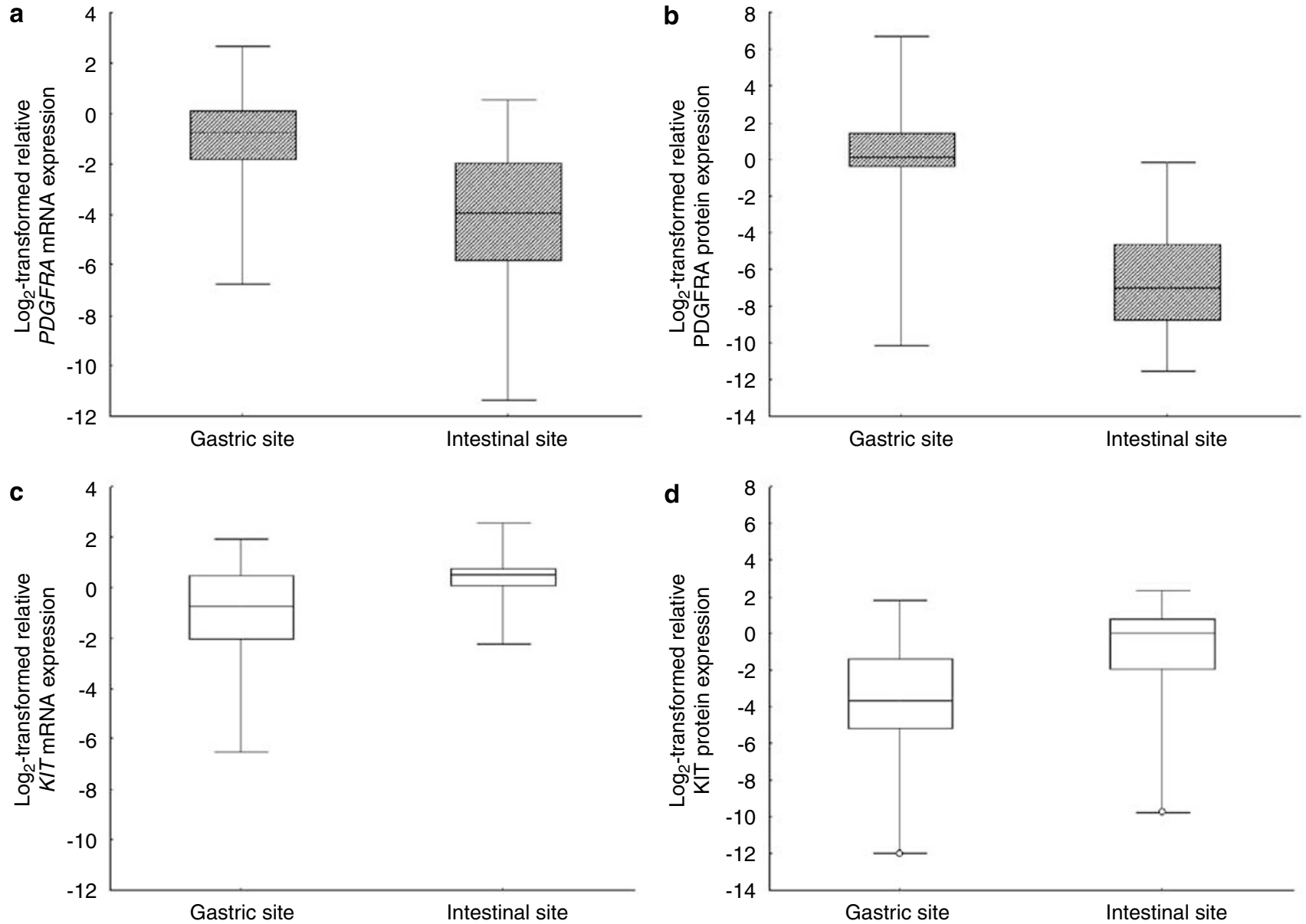

Figure 3 Site-dependent mRNA and protein expression of KIT and PDGFRA. (a) PDGFRA mRNA expression; (b) PDGFRA protein expression; (c) KIT mRNA expression; and (d) KIT protein expression. PDGFRA mRNA and protein expression (hatched box) were significantly higher in GISTs from gastric sites. KIT mRNA and protein expression (open box) were significantly higher in GISTs from intestinal sites. Horizontal line, median; inner box, 25 and 75\% interval; outer spread, minimum and maximum.

intestine. Furthermore, gastric GISTs had a 2.6-fold lower mRNA expression and a 5.3-fold lower protein expression of KIT compared to intestinal GISTs. As PDGFRA mutations were found exclusively in gastric GISTs, we performed a subgroup analysis among KIT-mutated GISTs to preclude a mutation-dependent bias. This analysis confirmed a further mutation-independent correlation between higher KIT expression and intestinal tumor site, and higher PDGFRA expression and gastric tumor site. Gastric GISTs with KIT mutation had a 5.5-fold higher mRNA expression and a 52.2-fold higher protein expression of PDGFRA compared to intestinal GISTs with KIT mutation. On the other hand, intestinal GISTs had a 3.7-fold higher protein expression of KIT. Therefore, a site-dependent constitutional difference in the expression of KIT and PDGFRA exists and is maintained irrespective of the type of activating mutation that may occur.

This new finding of a different site-dependent expression of KIT and PDGFRA is of further relevance for understanding the site-dependent differences in the clinical behavior of GISTs. Tumor site is an independent prognostic parameter in GISTs, with intestinal GISTs being more clinically aggressive compared to gastric GISTs. ${ }^{18,19}$ Although this clinical observation has been correlated with distinct gene expression profiles between gastric and intestinal GISTs ${ }^{20}$ it remains unclear whether this difference is already determined before neoplastic transformation, or is the consequence of a discrete genetic evolution of gastric and intestinal GISTs. GIST tumor cells are dependent on oncogenic KIT or PDGFRA signaling, permitting increased ligand-independent cell proliferation. ${ }^{4}$ Although secondary genetic events are doubtless involved in acceleration of malignant potential in GISTs, it is likely that the simple expression and activation level of KIT and PDGFRA at least initially controls the intensity of intracellular signal transduction, and, through the balance between cell proliferation and cell survival, the biological tumor behavior. Whereas the previously described mutation-dependent regulation of KIT and PDGFRA expression might account for the less aggressive clinical behavior of GISTs with PDGFRA mutation, ${ }^{11}$ the 

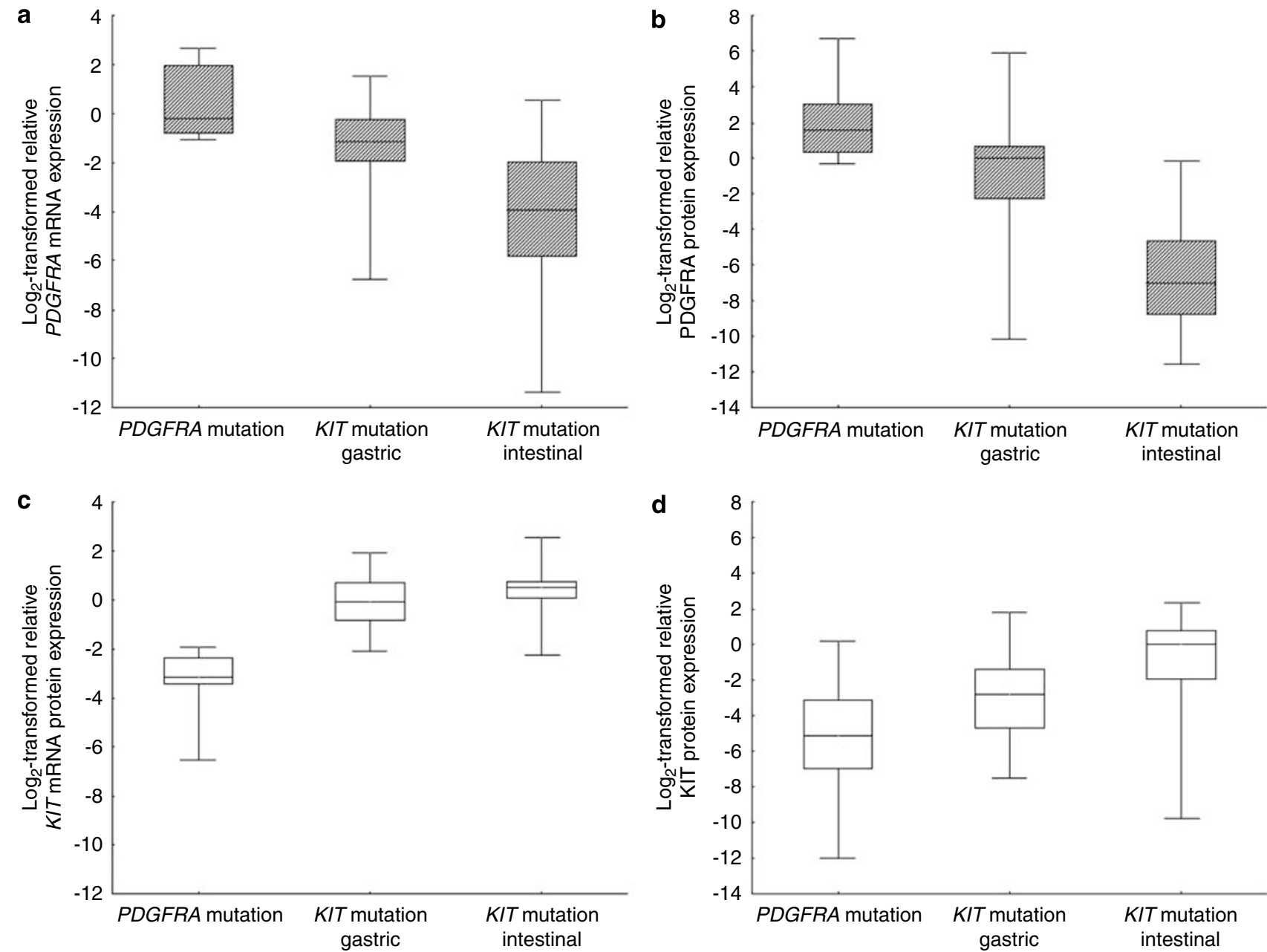

Figure 4 Comparison of mutation- and site-dependent effects on mRNA and protein expression of KIT and PDGFRA. (a) PDGFRA mRNA expression; (b) PDGFRA protein expression; (c) KIT mRNA expression; and (d) KIT protein expression. PDGFRA mRNA and protein expression (hatched box) were equally mutation- and site-dependent. KIT mRNA expression (open box) was strongly mutationdependent, but only slightly site-dependent. Horizontal line, median; inner box, 25 and 75\% interval; outer spread, minimum and maximum.

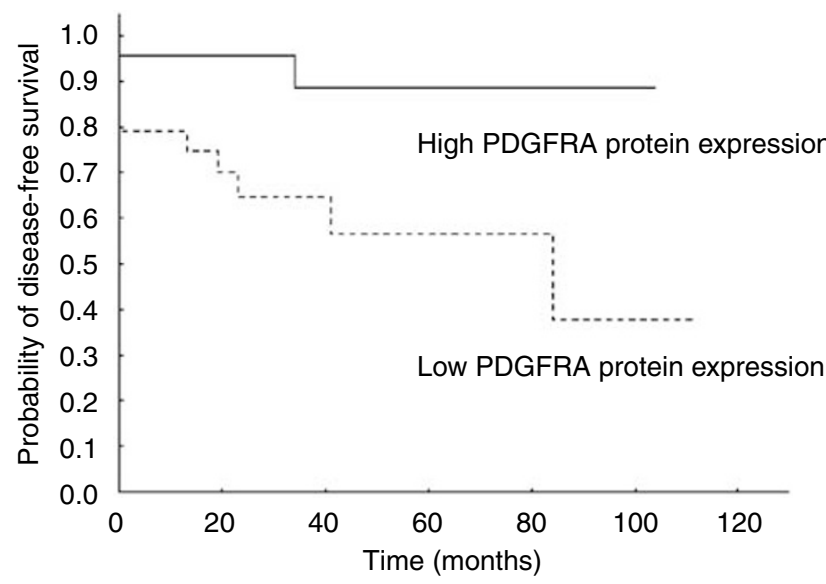

Figure 5 Expression of PDGFRA and disease-free survival. A twosided group-wise comparison of tumors with high (continuous line) vs low (dotted line) PDGFRA protein expression separated by the mean revealed that higher PDGFRA protein expression significantly correlated with longer disease-free survival $(P=0.01)$ current finding of a site-dependent regulation of KIT and PDGFRA expression provides a reason for the less aggressive clinical behavior of gastric GISTs. ${ }^{18}$

The current study cannot clarify whether KIT and PDGFRA influence each others' expression reciprocally or are regulated independently. Assuming the latter model, our finding would argue for a derivation of gastric and intestinal GISTs from distinct types of precursor cells, as has already been formulated by Nishitani et al. ${ }^{27}$ To date, the interstitial cells of Cajal located in the muscular layer of the gastrointestinal tract are regarded as the precursor cell for GISTs. Light and electron microscopy studies have demonstrated that there are morphologically distinguishable types of interstitial cells of Cajal with a site-specific distribution in the gastrointestinal tract. ${ }^{28} \mathrm{An}$ immunohistochemical analysis revealed that not all interstitial cells of Cajal in the human gut expressed KIT. ${ }^{29}$ The same authors reported that blocking of KIT signaling in newborn mice resulted in the almost complete disappearance 
of interstitial cells of Cajal, while the remaining undifferentiated precursor cells developed an ultrastructural smooth muscle cell phenotype. ${ }^{30}$ Thus a subset of interstitial cells of Cajal seems to be dependent on KIT signaling for the maintenance of their specific phenotype, while the role of PDGFRA signaling in these cells remains to be investigated. Taken together, a dependency of different interstitial cells of Cajal in the human gut on KIT or PDGFRA expression and signaling for maintenance of functionality is likely, and would explain the observed site-dependent differences in the expression of KIT and PDGFRA.

\section{Acknowledgement}

We thank Kristina Hänecke for excellent technical assistance.

\section{Disclosure/conflict of interest}

The authors state that there is no conflict of interest.

\section{References}

1 Hirota S, Isozaki K, Moriyama Y, et al. Gain-of-function mutations of c-kit in human gastrointestinal stromal tumors. Science 1998;279:577-580.

2 Heinrich MC, Corless CL, Duensing A, et al. PDGFRA activating mutations in gastrointestinal stromal tumors. Science 2003;299:708-710.

3 Sircar K, Hewlett BR, Huizinga JD, et al. Interstitial cells of Cajal as precursors of gastrointestinal stromal tumors. Am J Surg Pathol 1999;23:377-389.

4 Roskoski Jr R. Signaling by Kit protein-tyrosine kinase-the stem cell factor receptor. Biochem Biophys Res Commun 2005;337:1-13.

5 Heinrich MC, Corless CL, Demetri GD, et al. Kinase mutations and imatinib response in patients with metastatic gastrointestinal stromal tumor. J Clin Oncol 2003;21:4342-4349.

6 Corless CL, Fletcher JA, Heinrich MC. Biology of gastrointestinal stromal tumors. J Clin Oncol 2004;22:3813-3825.

7 Martin J, Poveda A, Llombart-Bosch A, et al. Deletions affecting codons $557-558$ of the c-KIT gene indicate a poor prognosis in patients with completely resected gastrointestinal stromal tumors: a study by the Spanish Group for Sarcoma Research (GEIS). J Clin Oncol 2005;23:6190-6198

8 Andersson J, Bumming P, Meis-Kindblom JM, et al. Gastrointestinal stromal tumors with KIT exon 11 deletions are associated with poor prognosis. Gastroenterology 2006;130:1573-1581.

9 Corless CL, Schroeder A, Griffith D, et al. PDGFRA mutations in gastrointestinal stromal tumors: frequency, spectrum and in vitro sensitivity to imatinib. J Clin Oncol 2005;23:5357-5364.

10 Wardelmann E, Neidt I, Bierhoff E, et al. c-kit mutations in gastrointestinal stromal tumors occur preferentially in the spindle rather than in the epithelioid cell variant. Mod Pathol 2002;15:125-136.
11 Lasota J, Dansonka-Mieszkowska A, Sobin LH, et al. A great majority of GISTs with PDGFRA mutations represent gastric tumors of low or no malignant potential. Lab Invest 2004;84:874-883.

12 Wardelmann E, Hrychyk A, Merkelbach-Bruse S, et al. Association of platelet-derived growth factor receptor alpha mutations with gastric primary site and epithelioid or mixed cell morphology in gastrointestinal stromal tumors. J Mol Diagn 2004;6: 197-204.

13 Wasag B, Debiec-Rychter M, Pauwels P, et al. Differential expression of KIT/PDGFRA mutant isoforms in epithelioid and mixed variants of gastrointestinal stromal tumors depends predominantly on the tumor site. Mod Pathol 2004;17:889-894.

14 Pauls K, Merkelbach-Bruse S, Thal D, et al. PDGFRalpha- and c-kit-mutated gastrointestinal stromal tumors (GISTs) are characterized by distinctive histological and immunohistochemical features. Histopathology 2005;46:166-175.

15 Penzel R, Aulmann S, Moock M, et al. The location of KIT and PDGFRA gene mutations in gastrointestinal stromal tumors is site and phenotype associated. J Clin Pathol 2005;58:634-639.

16 Subramanian S, West RB, Corless CL, et al. Gastrointestinal stromal tumors (GISTs) with KIT and PDGFRA mutations have distinct gene expression profiles. Oncogene 2004;23:7780-7790.

17 Kang HJ, Nam SW, Kim H, et al. Correlation of KIT and platelet-derived growth factor receptor alpha mutations with gene activation and expression profiles in gastrointestinal stromal tumors. Oncogene 2005;24:1066-1074.

18 Miettinen M, Sobin LH, Lasota J. Gastrointestinal stromal tumors of the stomach: a clinicopathologic, immunohistochemical, and molecular genetic study of 1765 cases with long-term follow-up. Am J Surg Pathol 2005;29:52-68.

19 Miettinen M, Makhlouf H, Sobin LH, et al. Gastrointestinal stromal tumors of the jejunum and ileum: a clinicopathologic, immunohistochemical, and molecular genetic study of 906 cases before imatinib with long-term follow-up. Am J Surg Pathol 2006;30:477489.

20 Antonescu CR, Viale A, Sarran L, et al. Gene expression in gastrointestinal stromal tumors is distinguished by KIT genotype and anatomic site. Clin Cancer Res 2004;10:3282-3290.

21 Fletcher CD, Berman JJ, Corless C, et al. Diagnosis of gastrointestinal stromal tumors: a consensus approach. Hum Pathol 2002;33:459-465.

22 Miettinen M, El Rifai W, Sobin HL, et al. Evaluation of malignancy and prognosis of gastrointestinal stromal tumors: a review. Hum Pathol 2002;33:478-483.

23 Haller F, Gunawan B, von Heydebreck A, et al. Prognostic role of E2F1 and members of the CDKN2A network in gastrointestinal stromal tumors. Clin Cancer Res 2005;11:6589-6597.

24 Pfaffl MW. A new mathematical model for relative quantification in real-time RT-PCR. Nucleic Acids Res 2001;29:e45.

25 Haller F, Kulle B, Schwager S, et al. Equivalence test in quantitative reverse transcription polymerase chain reaction: confirmation of reference genes suitable for normalization. Anal Biochem 2004;335:1-9.

26 Ihaka R, Gentleman RR. A language for data analysis and graphics. J Comp Graph Stat 1996;5:299-314. 
27 Nishitani A, Hirota S, Nishida T, et al. Differential expression of connexin 43 in gastrointestinal stromal tumors of gastric and small intestinal origin. J Pathol 2005;206:377-382.

28 Faussone-Pellegrini MS, Thuneberg L. Guide to the identification of interstitial cells of Cajal. Microsc Res Tech 1999;47:248-266.
29 Torihashi S, Horisawa M, Watanabe Y. c-Kit immunoreactive interstitial cells in the human gastrointestinal tract. J Auton Nerv Syst 1999;75:38-50.

30 Torihashi S, Nishi K, Tokutomi Y, et al. Blockade of kit signaling induces transdifferentiation of interstitial cells of cajal to a smooth muscle phenotype. Gastroenterology 1999;117:140-148. 